\title{
Discussion of a Possible Corrected Black Hole Entropy
}

\author{
Miao He, Ziliang Wang, Chao Fang, Daoquan Sun, and Jianbo Deng $\mathbb{D}$
}

Institute of Theoretical Physics, Lanzhou University, Lanzhou 730000, China

Correspondence should be addressed to Jianbo Deng; dengjb@lzu.edu.cn

Received 21 November 2017; Revised 31 January 2018; Accepted 12 February 2018; Published 11 March 2018

Academic Editor: Habib Mazharimousavi

Copyright (C) 2018 Miao He et al. This is an open access article distributed under the Creative Commons Attribution License, which permits unrestricted use, distribution, and reproduction in any medium, provided the original work is properly cited. The publication of this article was funded by SCOAP ${ }^{3}$.

\begin{abstract}
Einstein's equation could be interpreted as the first law of thermodynamics near the spherically symmetric horizon. Through recalling the Einstein gravity with a more general static spherical symmetric metric, we find that the entropy would have a correction in Einstein gravity. By using this method, we investigate the Eddington-inspired Born-Infeld (EiBI) gravity. Without matter field, we can also derive the first law in EiBI gravity. With an electromagnetic field, as the field equations have a more general spherically symmetric solution in EiBI gravity, we find that correction of the entropy could be generalized to EiBI gravity. Furthermore, we point out that the Einstein gravity and EiBI gravity might be equivalent on the event horizon. At last, under EiBI gravity with the electromagnetic field, a specific corrected entropy of black hole is given.
\end{abstract}

\section{Introduction}

Black hole thermodynamics has been proposed for many years since the entropy and temperature were found by Bekenstein and Hawking $[1,2]$, even getting many interesting results, like the four laws of black hole thermodynamics. It established the connection between the gravity and thermodynamics.

The entropy is assumed to be proportional to its horizon area [1], and it is well-known that the so-called area formula of black hole entropy holds only in Einstein gravity. However, when some higher order curvature terms appear in some gravity theory, the area formula has to be modified [3]. A logarithmic term often occurs in the correction like the black hole entropy in loop quantum gravity (quantum geometry) [4-6] and thermal equilibrium fluctuation $[7,8]$. The correction of entropy has been studied in Gauss-Bonnet gravity [9], Lovelock gravity [10], and $f(R)$ gravity [11]. In the apparent horizon of FRW universe, the entropy also has a correction [12].

Einstein's equation can be derived from the thermodynamics [13]; on the other side, the thermodynamic route to the gravity field equation, which could get the first law of thermodynamics in Einstein gravity, was proposed by Padmanabhan [14-16]. It indicated a generic connection between thermodynamics of horizons and gravity, although it is not yet understood at a deeper level [17]. This technique has been used in Gauss-Bonnet gravity and Lanczos-Lovelock gravity [15]; the corrected entropy is the same in $[18,19]$, respectively.

The EiBI gravity was inspired by Bañados and Ferreira [20]. It is completely equivalent to the Einstein gravity in vacuum, but in the presence of matter it would show many interesting results, like an alternative theory of Big Bang singularity in early universe [21] and the mass inflation in EiBI black holes [22, 23]. However, there are few investigations for the thermodynamic properties of black hole in EiBI gravity, as it has a complicated spherically symmetric solution when the electromagnetic field is considered [24].

In this paper, inspired by Padmanabhan [14-16], we derive the first law of black hole thermodynamics with the commonly accepted thermodynamics quantities from Einstein's equation. We also use this technique in EiBI gravity and get the known first law; the results show a more general formula of entropy, which also holds for AdS Schwarzschild black hole and AdS R-N black hole. Motived by this, supposing a more general static spherically symmetric metric, we get the same result in Einstein gravity.

This paper is organized as follows: In Section 2, there is a derivation of the thermodynamic identity from Einstein 
gravity; we also get a more general formula of entropy. In Section 3, we investigate the EiBI gravity by using the thermodynamic route to the field equation and get the formula of entropy in EiBI gravity. Conclusions and discussion are given in Section 4.

\section{Black Hole Thermodynamic Identity from Einstein's Equation}

Einstein's equation can be derived from the thermodynamics [13]. On the other side, it is possible to interpret Einstein's equation near the spherical symmetric event horizon as the first law of thermodynamics which was proposed by Padmanabhan [14-16]. However, the thermodynamic quantities might not be consistent with the normal ones, especially the pressure and internal energy. Fortunately, through restudying the field equation, we can also derive the first law of thermodynamics with the commonly accepted thermodynamic quantities $[25,26]$.

Considering a static spherically symmetric space-time

$$
d s^{2}=-f(r) d t^{2}+\frac{1}{f(r)} d r^{2}+r^{2}\left(d \theta^{2}+\sin ^{2} \theta d \phi^{2}\right)
$$

and the event horizon $r=r_{H}$ satisfying $f\left(r_{H}\right)=0$, then one can get its thermodynamic quantities

$$
\begin{aligned}
T & =\frac{\kappa}{2 \pi}=\frac{f^{\prime}\left(r_{H}\right)}{4 \pi}, \\
S & =\pi r_{H}^{2}, \\
V & =\frac{4 \pi}{3} r_{H}^{3} .
\end{aligned}
$$

If we consider AdS space-time with a negative cosmological constant $\Lambda$, there would be a pressure $P=-\Lambda / 8 \pi$ [25]. The mass of black hole was treated as enthalpy [26] and the first law of black hole thermodynamics is

$$
d H=d M=T d S+V d P .
$$

Through the Legendre transformation, one can get the internal energy

$$
d U=T d S-P d V .
$$

Particularly, the internal energies for AdS Schwarzschild black hole and AdS R-N black hole are

$$
\begin{aligned}
U_{\text {Schwarzschild }} & =\frac{r_{H}}{2}, \\
U_{\mathrm{R}-\mathrm{N}} & =\frac{r_{H}}{2}+\frac{Q^{2}}{2 r_{H}} .
\end{aligned}
$$

Einstein's equation with a cosmological constant is

$$
G_{\mu \nu}+\Lambda g_{\mu \nu}=8 \pi T_{\mu \nu} .
$$

If the metric has the form of (1), in the case of $T_{\mu \nu}=0$, one can obtain the $\theta \theta$ component of the equation

$$
-1+f(r)+r f^{\prime}(r)=-\Lambda r^{2}
$$

Setting $r=r_{H}$ and then multiplying the above equation by $d r_{H}$, we can rewrite (7) as

$$
\frac{f^{\prime}\left(r_{H}\right)}{4 \pi} d\left(\pi r_{H}^{2}\right)-d\left(\frac{r_{H}}{2}\right)=-\frac{\Lambda}{8 \pi} d\left(\frac{4 \pi r_{H}^{3}}{3}\right) .
$$

Noticing (2), the above equation can be regarded as the first law of black hole thermodynamics, since $U=r_{H} / 2$ for the Schwarzschild solution.

For a charged AdS black hole, the metric also takes the form of (1). The energy-momentum tensor of electromagnetic field is

$$
T_{\mu \nu}=\frac{1}{4 \pi}\left(F_{\mu \sigma} F_{v}^{\sigma}-\frac{1}{4} g_{\mu \nu} F_{\sigma \rho} F^{\sigma \rho}\right)
$$

Its nonzero components are

$$
\begin{aligned}
T_{t t} & =\frac{f E_{0}^{2}}{8 \pi}, \\
T_{r r} & =-\frac{f^{-1} E_{0}^{2}}{8 \pi}, \\
T_{\theta \theta} & =\frac{r^{2} E_{0}^{2}}{8 \pi}, \\
T_{\phi \phi} & =\frac{r^{2} \sin ^{2} \theta E_{0}^{2}}{8 \pi},
\end{aligned}
$$

where $E_{0}=Q / r^{2}$, and $Q$ represents the charge of black hole. According to Einstein's equations, one can also get

$$
-1+f(r)+r f^{\prime}(r)=-\Lambda r^{2}-r^{2} E_{0}^{2}
$$

By the same technique, and treating $Q$ as a constant, we get

$$
\frac{f^{\prime}\left(r_{H}\right)}{4 \pi} d\left(\pi r_{H}^{2}\right)-d\left(\frac{r_{H}}{2}+\frac{Q^{2}}{2 r_{H}}\right)=P d\left(\frac{4 \pi r_{H}^{3}}{3}\right),
$$

which can be also treated as the first law with the thermodynamic quantities in (2), and one can verify that $U=r_{H} / 2+$ $Q^{2} / 2 r_{H}$ for the AdS R-N black hole.

Here we keep $Q$ as a constant, which means a chargeless particle falls into the AdS R-N black hole; (12) is consistent with the first law. While a charged particle falls into the AdS R$\mathrm{N}$ black hole, the event horizon $r_{H}$ would arise due to changes of $d M$ and $d Q$; then (12) could be rewritten as [15]

$$
\begin{aligned}
& \frac{f^{\prime}\left(r_{H}\right)}{4 \pi} d\left(\pi r_{H}^{2}\right)-d\left(\frac{r_{H}}{2}+\frac{Q^{2}}{2 r_{H}}\right)+\frac{Q}{r_{H}} d Q \\
& =P d\left(\frac{4 \pi r_{H}^{3}}{3}\right) .
\end{aligned}
$$

Then it would adopt to the first law with the formulation

$$
d U=T d S-P d V+\Phi d Q .
$$

We should point out that $T_{\mu \nu}$ contributes to the internal energy $U$. 
Thus Einstein's equation can be interpreted as the first law of thermodynamic near the event horizon. The technique was first proposed by Padmanabhan, and some relevant comments about the meaning of thermodynamic quantities for this result were given [14-17]. Since we used the different thermodynamics quantities in our derivation compared with Padmanabhan's work, there are some comments we would like to add. Firstly, this method can be applied to single horizon and multiple horizons space-time, but this is just a local description of horizon thermodynamics which means the temperature, entropy, and energy are local quantities just for one horizon. In addition, this method still has some problem to solve, when it was applied to cosmic horizon or de Sitter horizon, because the definition of the temperatures for cosmic horizon is quite different. Secondly, it seems that there is a manifest arbitrariness or freedom in the derivations. One could multiply the entire equation by an arbitrary function; then the expressions for entropy, internal energy, and volume would be another one. In fact, if we consider the initial value, that is, $S=A / 4, V=4 \pi r_{H}^{3} / 3$, for Schwarzschild space-time, this problem would disappear. Finally, the perturbation of the static space-time can be interpreted in terms of the physical process of black hole evaporation or hawking radiation. One can also interpret the relations by, say, dropping test particles into the black hole.

Note that the structure of the equation itself allows us to read off the expression for entropy. This technique has been used for Gauss-Bonnet gravity and Lovelock gravity [15], in which their entropy formulas are the same as [18, 19], respectively. We would like to consider a more general static spherical symmetric metric

$$
\begin{aligned}
d s^{2}= & -\psi(r)^{2} f(r) d t^{2}+\frac{1}{f(r)} d r^{2} \\
& +r^{2}\left(d \theta^{2}+\sin ^{2} \theta d \phi^{2}\right) .
\end{aligned}
$$

One can calculate its nonzero components of Ricci tensor:

$$
\begin{aligned}
R_{t t}= & \frac{\left(\psi^{2} f\right)^{\prime \prime} f}{2}-\frac{\left(\psi^{2} f\right)^{\prime} f}{4}\left(-\frac{f^{\prime}}{f}+\frac{\left(\psi^{2} f\right)^{\prime}}{\psi^{2} f}\right) \\
& +\frac{\left(\psi^{2} f\right)^{\prime} f}{r}, \\
R_{r r}= & -\frac{\left(\psi^{2} f\right)^{\prime \prime}}{2 \psi^{2} f}+\frac{\left(\psi^{2} f\right)^{\prime}}{4 \psi^{2} f}\left(-\frac{f^{\prime}}{f}+\frac{\left(\psi^{2} f\right)^{\prime}}{\psi^{2} f}\right)-\frac{f^{\prime}}{r f}, \\
R_{\theta \theta}= & 1-\frac{r f}{2}\left(\frac{f^{\prime}}{f}+\frac{\left(\psi^{2} f\right)^{\prime}}{\psi^{2} f}\right)-f, \\
R_{\phi \phi}= & \sin ^{2} \theta R_{\theta \theta} .
\end{aligned}
$$

And Einstein's equation with a cosmological constant can be written as

$$
R_{\mu \nu}-\Lambda g_{\mu \nu}=8 \pi\left(T_{\mu \nu}-\frac{1}{2} T g_{\mu \nu}\right)
$$

For the Schwarzschild vacuum $T_{\mu \nu}=0$, one can get $\psi=C$, a constant. We can always have $\psi=1$ by choosing the coordinate time $d \vec{t}=C d t$ without changing the killing vector; then the metric would return back to the spherically symmetric metric (1). For the charged black hole, we have got the Reissner-Nordstrom metric, which also implies $\psi=1$; thus the first law could be obtained. The reason of this result might be that the matter field leads to $\psi=1$.

To get a general case, we just consider the $\theta \theta$ component of (17), which can be expressed as

$$
\begin{aligned}
1 & -\frac{r f(r)}{2}\left(\frac{f^{\prime}(r)}{f(r)}+\frac{\left(\psi^{2} f(r)\right)^{\prime}}{\psi^{2} f(r)}\right)-f(r) \\
= & \Lambda r^{2}+8 \pi\left(T_{\theta \theta}-\frac{1}{2} T r^{2}\right) .
\end{aligned}
$$

We assume the metric satisfies $f\left(r_{H}\right)=0$ and $\psi\left(r_{H}\right) \neq 0$ on the event horizon. By setting $r=r_{H}$ and considering the matter field contributes to the internal energy $U$ and then multiplying $d r_{H}$ one can get

$$
d U-\frac{r_{H}}{2} f^{\prime}\left(r_{H}\right) d r_{H}=-P d\left(\frac{4 \pi r_{H}^{3}}{3}\right),
$$

where

$$
d U=\left[\frac{1}{2}+4 \pi\left(T_{\theta \theta}-\frac{1}{2} T r_{H}^{2}\right)\right] d r_{H} .
$$

One can verify that for the AdS Schwarzschild black hole, it reduces to $U=r_{H} / 2$ and for the AdS R-N black hole it gives $U=r_{H} / 2+Q^{2} / 2 r_{H}$. So the internal energy expression is just a generalization.

The Hawking temperature on the event horizon becomes

$$
T=\frac{\kappa}{2 \pi}=\frac{\psi\left(r_{H}\right) f^{\prime}\left(r_{H}\right)}{4 \pi} .
$$

Now rewrite (19) as

$$
d U-T\left(\frac{2 \pi r_{H}}{\psi\left(r_{H}\right)} d r_{H}\right)=-P d V .
$$

One would find the entropy has to satisfy

$$
d S=\frac{2 \pi r_{H}}{\psi\left(r_{H}\right)} d r_{H}
$$

or

$$
S=\int \frac{2 \pi r_{H}}{\psi\left(r_{H}\right)} d r_{H}
$$

Thus, we generalize the corrected entropy formula to Einstein gravity for static spherically symmetric metric equation (15). Once $\psi=1$, it is obvious that $S=\pi r_{H}^{2}=A / 4$, which is the well-known Bekenstein-Hawking black hole entropy. For the Schwarzschild black hole and R-N black hole, which all have $\psi=1, S=A / 4$. 
In fact, Matt Visser has proposed that the entropy of "dirty" black holes might not equal quarter of area of event horizon [27, 28]. Generically, a "dirty" black hole is a black hole with various classical matter fields, higher curvature terms in the gravity Lagrangian, or some other versions of quantum hair. For the Einstein gravity, a more general metric (15) could be caused by some special matter fields like electromagnetism with Dilaton fields [29]. So the entropy also should be corrected in Einstein gravity. In the next section, we would like to consider the EiBI gravity [20]; its Lagrangian includes the higher curvature terms in (25), which could be interpreted as the self-gravity; we will show our discussion of this method in the Eddington-inspired Born-Infeld gravity and get its thermodynamic quantities.

\section{The Entropy in Eddington-Inspired Born-Infeld Gravity}

The Eddington-inspired Born-Infeld theory of gravity is based on the Palatini formulation which treats the metric and connection as independent fields [20]. Its action can be written as

$$
\begin{aligned}
S= & \frac{1}{8 \pi \kappa} \int d^{4} x\left[\sqrt{\left|g_{\mu \nu}+\kappa R_{\mu \nu}(\Gamma)\right|}-\lambda \sqrt{g}\right] \\
& +S_{M}(g, \Gamma, \Psi)
\end{aligned}
$$

where $g_{\mu \nu}$ is the metric of space-time and its determinant is $g, R_{\mu \nu}$ is the symmetric Ricci tensor related to $\Gamma$, the dimensionless parameter $\lambda=1+\kappa \Lambda$, and the parameter $\kappa$ has the inverse dimension of cosmological constant $\Lambda$.

By varying the action with respect to $g_{\mu \nu}$ and $\Gamma$, one obtains the equation of motion:

$$
\begin{aligned}
q_{\mu \nu} & =g_{\mu \nu}+\kappa R_{\mu \nu} \\
\sqrt{|q|} q^{\mu \nu} & =\lambda \sqrt{|g|} g^{\mu \nu}-8 \pi \kappa \sqrt{|g|} T^{\mu \nu}
\end{aligned}
$$

where $q_{\mu \nu}$ is the auxiliary metric compatible with the connection to

$$
\Gamma_{\mu \nu}^{\lambda}=\frac{1}{2} q^{\lambda \sigma}\left(q_{\mu \sigma, \nu}+q_{\nu \sigma, \mu}-q_{\mu \nu, \sigma}\right)
$$

By combining (26) and (27) and then expanding the field equations to 2 nd order of $\kappa$ [20]

$$
\begin{aligned}
R_{\mu \nu} \simeq & \Lambda g_{\mu \nu}+8 \pi\left(T_{\mu \nu}-\frac{1}{2} T g_{\mu \nu}\right) \\
& +8 \pi \kappa\left[S_{\mu \nu}-\frac{1}{4} S g_{\mu \nu}\right]
\end{aligned}
$$

where $S_{\mu \nu}=T_{\mu}^{\alpha} T_{\alpha \nu}-(1 / 2) T T_{\mu \nu}$, one can find that the equation is the 1st-order correction to Einstein's equation. On the other hand, EiBI gravity can be interpreted as a correction of the matter term compared with Einstein gravity. Even the EiBI gravity is fully equivalent to the Einstein gravity in vacuum.
Let us consider the thermodynamics from the field equation in this gravity model. Generally, a static spherically symmetric metric $g_{\mu \nu}$ could be

$$
\begin{aligned}
d s_{g}^{2}= & -\psi^{2}(r) f(r) d t^{2}+\frac{1}{f(r)} d r^{2} \\
& +r^{2}\left(d \theta^{2}+\sin ^{2} \theta d \phi^{2}\right)
\end{aligned}
$$

and the auxiliary metric $q_{\mu \nu}$ is assumed as [24]

$$
\begin{aligned}
d s_{q}^{2}= & -G^{2}(r) F(r) d t^{2}+\frac{1}{F(r)} d r^{2} \\
& +H^{2}(r)\left(d \theta^{2}+\sin ^{2} \theta d \phi^{2}\right) .
\end{aligned}
$$

The Ricci tensor was calculated as follows:

$$
\begin{aligned}
R_{t t}= & 2 \frac{G G^{\prime} H^{\prime} F^{2}}{H}+\frac{G^{2} F F^{\prime} H^{\prime}}{H}+\frac{3}{2} G G^{\prime} F F^{\prime}+G G^{\prime \prime} F^{2} \\
& +\frac{1}{2} G^{2} F F^{\prime \prime}, \\
R_{r r}= & -2 \frac{H^{\prime \prime}}{H}-\frac{F^{\prime} H^{\prime}}{F H}-\frac{3}{2} \frac{G^{\prime} F^{\prime}}{G F}-\frac{G^{\prime \prime}}{G}-\frac{F^{\prime \prime}}{2 F}, \\
R_{\theta \theta}= & 1-H H^{\prime} F^{\prime}-\frac{G^{\prime}}{G} H H^{\prime} F-H^{\prime 2} F-H H^{\prime \prime} F, \\
R_{\phi \phi}= & \sin ^{2} \theta R_{\theta \theta},
\end{aligned}
$$

where $X^{\prime}=\partial X / \partial r$, which we will use in the rest of this paper.

Without the matter fields, (27) reduces to

$$
\begin{aligned}
\frac{H^{2}}{G F} & =\frac{\lambda r^{2}}{\psi f}, \\
G H^{2} F & =\lambda r^{2} \psi f, \\
G & =\lambda \psi,
\end{aligned}
$$

and thus one can obtain

$$
\begin{aligned}
G & =\lambda \psi, \\
F & =\lambda^{-1} f, \\
H^{2} & =\lambda r^{2} .
\end{aligned}
$$

Plugging these into (26), then the $\theta \theta$ component is

$$
1-r f^{\prime}(r)-\left(\frac{r \psi^{\prime}}{\psi}+1\right) f(r)=\frac{1}{\kappa}(\lambda-1) r^{2} .
$$

Near the event horizon $r=r_{H}\left(f\left(r_{H}\right)=0\right)$, we have

$$
d\left(\frac{r_{H}}{2}\right)-\frac{f^{\prime}\left(r_{H}\right)}{4 \pi} d\left(\pi r_{H}^{2}\right)=-P d\left(\frac{4 \pi r_{H}^{3}}{3}\right) .
$$

As the EiBI gravity is fully equivalent to the Einstein gravity in vacuum [20], the above equation could imply the first law. In 
fact, the black hole solution to EiBI gravity with no source is the same as Schwarzschild-de Sitter metric, which illustrates $\psi=1$ and $f=1-2 m / r+\Lambda r^{2} / 3$ [20]. The thermodynamic quantities in (36) also hold in EiBI gravity. Thus the first law can also be got from the EiBI gravity. Next, we would consider the EiBI gravity with electromagnetic field.

The energy-momentum tensor of electromagnetic field could be

$$
\begin{aligned}
T^{t t} & =\frac{\left(\psi^{2} f\right)^{-1} E_{0}^{2}}{8 \pi}, \\
T^{r r} & =-\frac{f E_{0}^{2}}{8 \pi}, \\
T^{\theta \theta} & =\frac{r^{-2} E_{0}^{2}}{8 \pi}, \\
T^{\phi \phi} & =\frac{r^{-2} \sin ^{-2} \theta E_{0}^{2}}{8 \pi},
\end{aligned}
$$

where $E_{0}=Q / r^{2}$ and $Q$ represents the charge of black hole. Then (27) becomes

$$
\begin{aligned}
\frac{H^{2}}{G F} & =\left(\lambda+\kappa E_{0}^{2}\right) \frac{r^{2}}{\psi f}, \\
G H^{2} F & =\left(\lambda+\kappa E_{0}^{2}\right) r^{2} \psi f, \\
G & =\left(\lambda-\kappa E_{0}^{2}\right) \psi,
\end{aligned}
$$

and one can get

$$
\begin{aligned}
G & =\left(\lambda-\kappa E_{0}^{2}\right) \psi, \\
F & =\left(\lambda-\kappa E_{0}^{2}\right)^{-1} f, \\
H^{2} & =\left(\lambda+\kappa E_{0}^{2}\right) r^{2} .
\end{aligned}
$$

The $\theta \theta$ component of (26) is written as

$$
\begin{aligned}
1- & \frac{\lambda+\kappa E_{0}^{2}+\kappa r E_{0} E_{0}^{\prime}}{\lambda-\kappa E_{0}^{2}} \cdot r f^{\prime}-\frac{Y}{\lambda-\kappa E_{0}^{2}} f \\
& =\frac{1}{\kappa}(\lambda-1) r^{2}+E_{0}^{2} r^{2},
\end{aligned}
$$

where

$$
Y=2 \kappa r E_{0} E_{0}^{\prime}+\frac{G^{\prime}}{G} H H^{\prime}-H^{\prime 2} .
$$

If we assume that the event horizon satisfies $f\left(r_{H}\right)=0$ and $\psi\left(r_{H}\right) \neq 0$, then set $r=r_{H}$ in (40) and multiply it by $d r_{H}$; noting $E_{0}^{\prime}=-2 E_{0} / r$, it gives

$$
d\left(\frac{r_{H}}{2}+\frac{Q^{2}}{2 r_{H}}\right)-\frac{r_{H}}{2} f^{\prime}\left(r_{H}\right) d r_{H}=-P d\left(\frac{4 \pi r_{H}^{3}}{3}\right) \text {. }
$$

This equation should be the first law since it can go back to (36) when $Q=0$. And one can also confirm this by noticing that $d U=d\left(r_{H} / 2+Q^{2} / 2 r_{H}\right)$ and $d V=d\left(4 \pi r_{H}^{3} / 3\right)$. Moreover, it gives the same result in Einstein gravity. So we should identify that

$$
T d S=\frac{r_{H}}{2} f^{\prime}\left(r_{H}\right) d r_{H}
$$

However, as the metric takes the form of (30), the surface gravity could be [29]

$$
\kappa=\lim _{r \rightarrow r_{H}} \frac{1}{2} \frac{\partial_{r} g_{t t}}{\sqrt{g_{t t} g_{r r}}}=\frac{\psi\left(r_{H}\right) f^{\prime}\left(r_{H}\right)}{2} .
$$

One can obtain the temperature on the event horizon

$$
T=\frac{\psi\left(r_{H}\right) f^{\prime}\left(r_{H}\right)}{4 \pi} .
$$

Then (43) would imply that

$$
d S=\left(\frac{2 \pi r_{H}}{\psi\left(r_{H}\right)}\right) d r_{H},
$$

or

$$
S=\int \frac{2 \pi r_{H}}{\psi\left(r_{H}\right)} d r_{H}
$$

Obviously, when $\psi=1$, one can get $S=\pi r_{H}^{2}$.

Thus, we get the entropy formula in EiBI gravity. Surprisingly, we find it also holds in Einstein gravity once metric takes the form of (30). Therefore, we can also get the first law for a more general static spherically symmetric metric in EiBI gravity. Moreover, one can find that (12) is the same as (42). It implies that the Einstein gravity and EiBI gravity might be equivalent on the event horizon from the view of black hole thermodynamics.

In fact, the black hole solution with electromagnetic field in EiBI gravity has been found, while $f\left(r_{H}\right)=0$ and $\psi\left(r_{H}\right) \neq$ $0[20,24]$. It is given as follows:

$$
\psi(r)=\frac{\sqrt{\lambda} r^{2}}{\sqrt{\lambda r^{4}+\kappa Q^{2}}} .
$$

With this result we can get the corrected entropy

$$
\begin{aligned}
S= & \int \frac{2 \pi r_{H}}{\psi\left(r_{H}\right)} d r_{H}=\pi \int \frac{1}{r_{H}^{2}} \sqrt{r_{H}^{4}+\frac{\kappa}{\lambda} Q^{2}} d r_{H}^{2} \\
= & \pi \sqrt{r_{H}^{4}+\frac{\kappa}{\lambda} Q^{2}}-\pi \sqrt{\frac{\kappa}{\lambda}}|Q| \\
& \cdot \ln \left(\sqrt{\frac{\kappa}{\lambda} \frac{|Q|}{r_{H}^{2}}}+\sqrt{1+\frac{\kappa}{\lambda} \frac{Q^{2}}{r_{H}^{4}}}\right) .
\end{aligned}
$$

A logarithmic term occurs in this formula as a corrected entropy. When $Q=0$, one gets $S=\pi r_{H}^{2}$, which is consistent with the vacuum case, and so it is the same as Einstein gravity. When $\kappa \rightarrow 0$, EiBI gravity would reduce to the Einstein gravity, and entropy becomes the Bekenstein-Hawking one. 


\section{Conclusion and Discussion}

In this paper, we restudied Padmanabhan's work that it is possible to write Einstein's equation for spherically symmetric space-time in the form of the first law of thermodynamics [14-17], but the thermodynamic quantities might not be consistent with the normal ones, especially the pressure and internal energy. By using this technique, we reproduced the first law with the commonly accepted thermodynamic quantities in the AdS space-time, and this technique provides an effective approach to read off the thermodynamic quantities. Next, we investigated a more general static spherically symmetric metric taking form (15) in Einstein gravity. It is surprising to find that the entropy might have a correction in Einstein gravity.

Since it provided a convenient approach to study the black hole thermodynamics just from the field equation, we investigated the black hole thermodynamic in EiBI gravity. We found that there is nothing different from Einstein gravity in vacuum, but entropy could be different from the R-N black hole when the electromagnetic field was considered. The corrected entropy from EiBI gravity should be (47), which can reduce to the Bekenstein-Hawking entropy when $\psi=1$, and it is also the same as Einstein gravity when $Q=0$ without the matter field [20]. Thus, the corrected entropy in Einstein gravity could be generalized to EiBI gravity.

Moreover, as the Einstein gravity and EiBI gravity hold the same result, we remarked that these two theories of gravity could be equivalent on the event horizon from the view of thermodynamics.

At last, as an example, a specific corrected entropy of the charged black hole in EiBI gravity was given. The entropy form would lead to something different for the black hole thermodynamics in EiBI gravity, like the phase transition and critical phenomenon. These would be left for our further research.

\section{Conflicts of Interest}

The authors declare that there are no conflicts of interest regarding the publication of this paper.

\section{Acknowledgments}

The authors would like to acknowledge the National Natural Science Foundation of China (Grant no. 11571342) for supporting them in this work.

\section{References}

[1] J. D. Bekenstein, "Black holes and entropy," Physical Review D: Particles, Fields, Gravitation and Cosmology, vol. 7, no. 8, pp. 2333-2346, 1973.

[2] S. W. Hawking, "Particle creation by black holes," Communications in Mathematical Physics, vol. 43, no. 3, pp. 199-220, 1975.

[3] R. M. Wald, "Black hole entropy is the Noether charge," Physical Review D: Particles, Fields, Gravitation and Cosmology, vol. 48, no. 8, pp. R3427-R3431, 1993.
[4] C. Rovelli, "Black hole entropy from loop quantum gravity," Physical Review Letters, vol. 77, no. 16, pp. 3288-3291, 1996.

[5] A. Ashtekar, J. Baez, A. Corichi, and K. Krasnov, "Quantum geometry and black hole entropy," Physical Review Letters, vol. 80, no. 5, pp. 904-907, 1998.

[6] R. K. Kaul and P. Majumdar, "Logarithmic correction to the Bekenstein-Hawking entropy," Physical Review Letters, vol. 84, no. 23, pp. 5255-5257, 2000.

[7] S. Das, P. Majumdar, and R. K. Bhaduri, "General logarithmic corrections to black-hole entropy," Classical and Quantum Gravity, vol. 19, no. 9, pp. 2355-2367, 2002.

[8] A. Chatterjee and P. Majumdar, "Universal canonical black hole entropy," Physical Review Letters, vol. 92, no. 14, article 141301, 2004.

[9] M. Cvetič, S. Nojiri, and S. D. Odintsov, "Black hole thermodynamics and negative entropy in de Sitter and anti-de Sitter Einstein-Gauss-Bonnet gravity," Nuclear Physics B, vol. 628, no. 1-2, pp. 295-330, 2002.

[10] R. Banerjee and S. K. Modak, "Quantum tunneling, blackbody spectrum and non-logarithmic entropy correction for Lovelock black holes," Journal of High Energy Physics, vol. 2009, no. 11, article 073, 2009.

[11] M. Akbar and R.-G. Cai, "Thermodynamic behavior of field equations for gravity," Physics Letters B, vol. 648, no. 2-3, pp. 243-248, 2007.

[12] R.-G. Cai, L.-M. Cao, and Y.-P. Hu, "Corrected entropy-area relation and modified Friedmann equations," Journal of High Energy Physics, vol. 2008, no. 8, article no. 090, 2008.

[13] T. Jacobson, "Thermodynamics of spacetime: the Einstein equation of state," Physical Review Letters, vol. 75, no. 7, pp. 1260-1263, 1995.

[14] D. Kothawala, S. Sarkar, and T. Padmanabhan, "Einstein's equations as a thermodynamic identity: The cases of stationary axisymmetric horizons and evolving spherically symmetric horizons," Physics Letters B, vol. 652, no. 5-6, pp. 338-342, 2007.

[15] A. Paranjape, S. Sarkar, and T. Padmanabhan, "Thermodynamic route to field equations in Lanczos-Lovelock gravity," Physical Review D: Particles, Fields, Gravitation and Cosmology, vol. 74, no. 10, Article ID 104015, 2006.

[16] T. Padmanabhan, "Classical and quantum thermodynamics of horizons in spherically symmetric spacetimes," Classical and Quantum Gravity, vol. 19, no. 21, pp. 5387-5408, 2002.

[17] T. Padmanabhan, "Gravity and the thermodynamics of horizons," Physics Reports, vol. 406, no. 2, pp. 49-125, 2005.

[18] R. C. Myers and J. Z. Simon, "Black-hole thermodynamics in Lovelock gravity," Physical Review D: Particles, Fields, Gravitation and Cosmology, vol. 38, no. 8, pp. 2434-2444, 1988.

[19] R.-G. Cai, "Gauss-Bonnet black holes in AdS spaces," Physical Review D: Particles, Fields, Gravitation and Cosmology, vol. 65, no. 8, Article ID 084014, 2002.

[20] M. Bañados and P. G. Ferreira, "Eddington's theory of gravity and its progeny," Physical Review Letters, vol. 105, no. 1, article 011101, 2010.

[21] P. P. Avelino and R. Z. Ferreira, "Bouncing Eddington-inspired Born-Infeld cosmologies: An alternative to inflation?” Physical Review D: Particles, Fields, Gravitation and Cosmology, vol. 86, no. 4, Article ID 041501, 2012.

[22] P. P. Avelino, "Inner structure of black holes in Eddingtoninspired Born-Infeld gravity: The role of mass inflation," Physical Review D: Particles, Fields, Gravitation and Cosmology, vol. 93, no. 4, Article ID 044067, 2016. 
[23] P. P. Avelino, "Mass inflation in Eddington-inspired Born-Infeld black holes: Analytical scaling solutions," Physical Review D: Particles, Fields, Gravitation and Cosmology, vol. 93, no. 10, Article ID 104054, 2016.

[24] S.-W. Wei, K. Yang, and Y.-X. Liu, "Black hole solution and strong gravitational lensing in Eddington-inspired Born-Infeld gravity," The European Physical Journal C, vol. 75, no. 6, article no. 253, 2015.

[25] B. P. Dolan, "The cosmological constant and black-hole thermodynamic potentials," Classical and Quantum Gravity, vol. 28, no. 12, Article ID 125020, 2011.

[26] D. Kastor, S. Ray, and J. Traschen, "Enthalpy and the mechanics of AdS black holes," Classical and Quantum Gravity, vol. 26, no. 19, Article ID 195011, 2009.

[27] M. Visser, "Dirty black holes: Entropy as a surface term," Physical Review D: Particles, Fields, Gravitation and Cosmology, vol. 48, no. 12, pp. 5697-5705, 1993.

[28] M. Visser, "Dirty black holes: entropy versus area," Physical Review D: Particles, Fields, Gravitation and Cosmology, vol. 48, no. 2, pp. 583-591, 1993.

[29] M. Visser, "Dirty black holes: thermodynamics and horizon structure," Physical Review D: Particles, Fields, Gravitation and Cosmology, vol. 46, no. 6, pp. 2445-2451, 1992. 

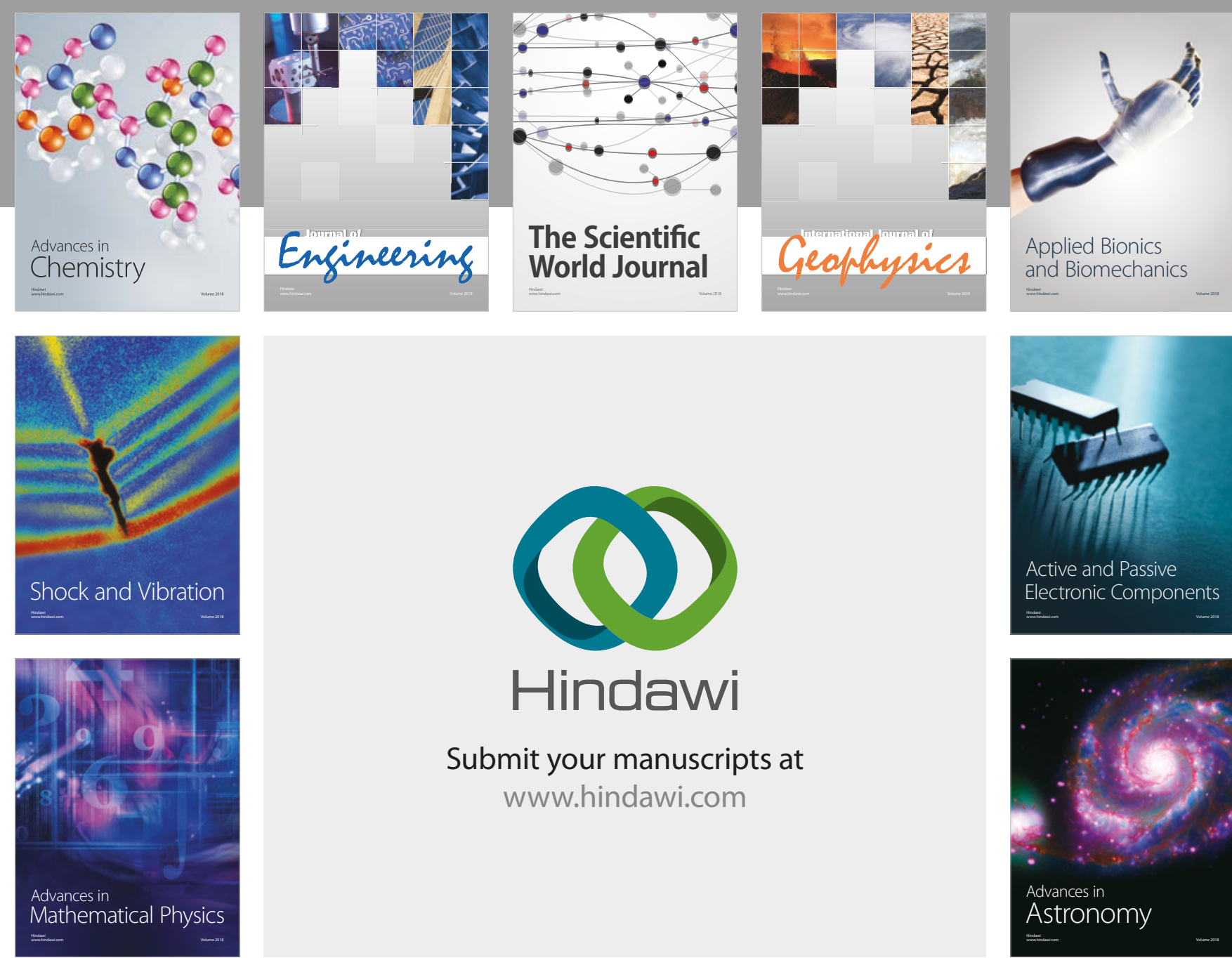

Submit your manuscripts at

www.hindawi.com

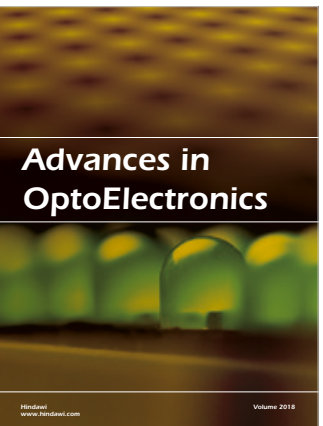

\section{Rotcting Machinery}
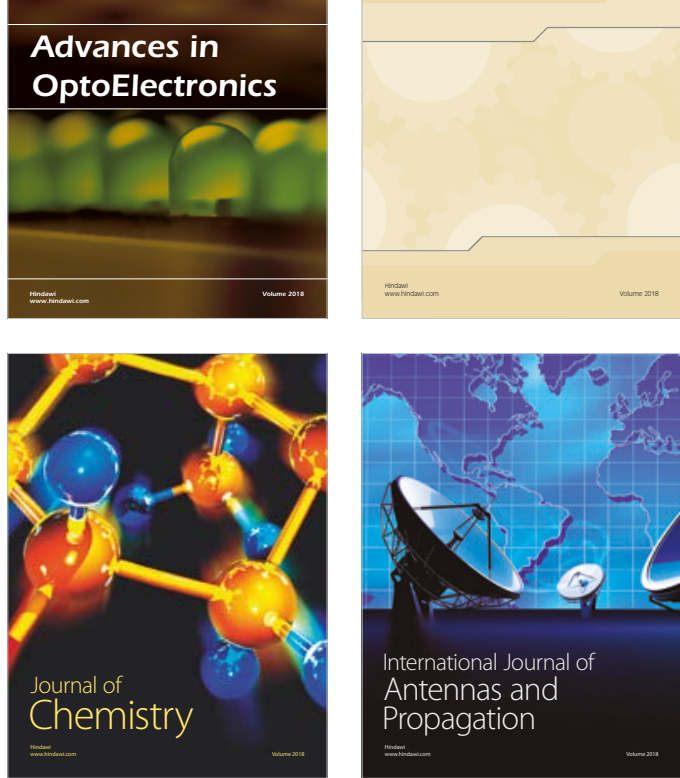

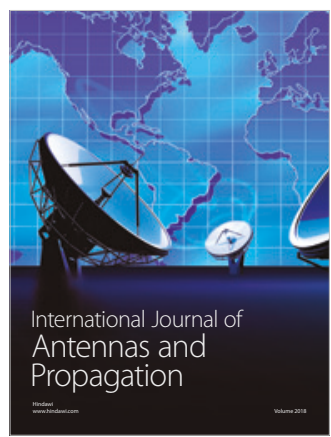

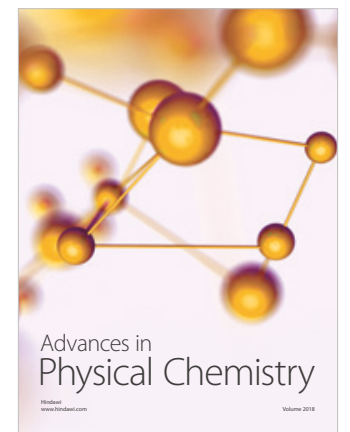

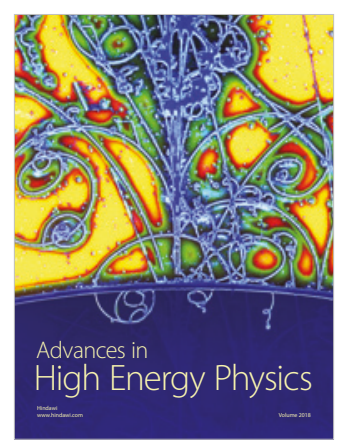

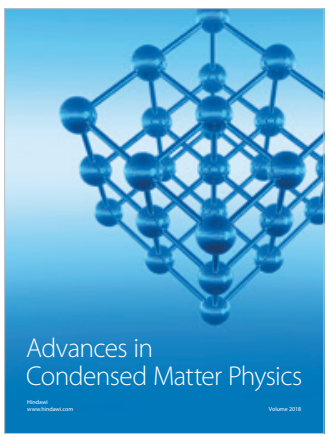

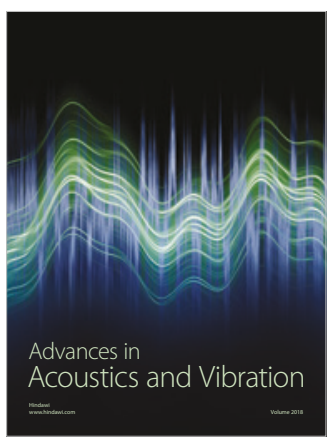

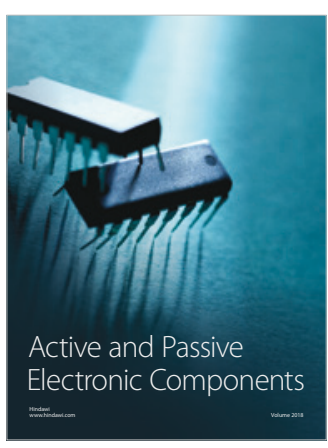
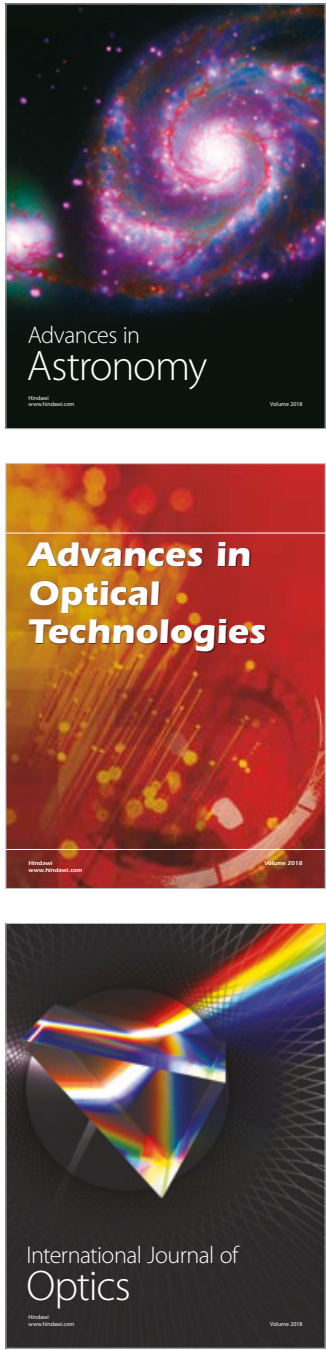